\title{
Gradual reactivation of vascular angiotensin I to angiotensin II conversion during chronic ACE inhibitor therapy in patients with diabetes mellitus
}

\author{
D. C. Sharman • A. D. Morris • A. D. Struthers
}

Received: 16 March 2007 / Accepted: 14 May 2007 /Published online: 4 August 2007

(C) Springer-Verlag 2007

\begin{abstract}
Aims/hypothesis In chronic heart failure there is gradual reactivation of vascular tissue angiotensin I (AI) to angiotensin II (AII) conversion over time in patients taking chronic ACE inhibitor therapy. However, it remains unknown whether the same overall phenomenon occurs in other patients taking chronic ACE inhibitor therapy, such as patients with type 2 diabetes mellitus.

Methods We studied 30 patients with type 2 diabetes mellitus (mean age $43.5 \pm 10.8$ years), all of whom received lisinopril $(20 \mathrm{mg} /$ day $)$ as part of their normal treatment. Over the course of the 18 month study, we made measurements at 0,9 and 18 months. These measurements included plasma values for components of the renin-angiotensinaldosterone system. In addition, we infused AI and AII into the brachial arteries of patients to assess vascular tissue AI to AII conversion.

Results There were no significant changes in plasma renin activity, ACE, AI, AII or aldosterone during the study. In contrast, vascular AI to AII conversion was significantly ( $p=$ 0.01 ) greater at 18 months than at 0 months. There was no change over time in the response to infused AII.

Conclusions/interpretation We have shown in vivo that vascular tissue AI to AII conversion gradually increases over time in patients with type 2 diabetes being treated with lisinopril. Further studies are required to determine whether this reactivation detracts from the cardioprotective effects of chronic ACE inhibitor therapy in diabetic patients, and if so, how best to overcome it.
\end{abstract}

D. C. Sharman · A. D. Morris · A. D. Struthers $(\square)$

Division of Medicine and Therapeutics, University of Dundee,

Ninewells Hospital and Medical School,

Dundee DD1 9SY, UK

e-mail: a.d.struthers@dundee.ac.uk
Keywords Cardiac complications · Clinical ·

Complications (all) · Endothelium · Hormones · Action ·

Human · Hypertension · Macrovascular disease .

Microvascular disease

\begin{tabular}{ll}
\multicolumn{2}{l}{ Abbreviations } \\
AI & $\begin{array}{l}\text { angiotensin I } \\
\text { AII }\end{array}$ \\
angiotensin II \\
ARB & angiotensin receptor blocker \\
BNF & British National Formulary \\
CHF & chronic heart failure \\
FBF & forearm blood flow \\
HOPE & Heart Outcomes Prevention Study \\
RAAS & renin-angiotensin-aldosterone system \\
VALIANT & Valsartan in Acute Myocardial Infarction Trial
\end{tabular}

\section{Introduction}

ACE inhibitors were first introduced for the treatment of hypertension and heart failure where their benefits were clearly established. Subsequently they were investigated in high-risk vascular patients such as in the Heart Outcomes Prevention Study (HOPE), where they were also found to reduce cardiovascular mortality [1]. These benefits were particularly strong in the diabetic subgroup of the HOPE study [2]. A similar pattern was seen in the Losartan Intervention for Endpoint Reduction in Hypertension study, in that the benefits of angiotensin II (AII) blockade over atenolol were particularly strong in the diabetic subgroup [3].

However, the overall benefit of ACE inhibitors in studies such as the HOPE study may be smaller than it could be, since the renin-angiotensin-aldosterone system (RAAS) appears, in some circumstances, to reactivate during 
chronic ACE inhibitor therapy. This reactivation phenomenon was first seen for plasma AII and aldosterone in patients with chronic heart failure (CHF) [4]. However, plasma levels are not that informative because there are two different components to the RAAS: circulating RAAS and tissue RAAS, with several lines of evidence suggesting that the former and the latter are often dissociated from each other [5-7]. Three recent studies in CHF have assessed whether vascular tissue RAAS still generates AII during chronic ACE inhibitor therapy. Two of these were in vivo studies, the other was an ex vivo study. All three showed that vascular tissue did indeed generate AII formation during chronic ACE inhibitor therapy [8-10]. In fact, one study showed that this tissue reactivation was a gradual process over time during chronic ACE inhibitor therapy [9]. All three of the studies mentioned were performed in CHF patients [8-10].

As yet, it remains unknown whether this same reactivation of vascular tissue RAAS occurs in other diseases where chronic ACE inhibitor therapy is used, such as the kind of type 2 diabetic patients studied in the micro HOPE study. Neuroendocrine activation is known to be particularly strong in $\mathrm{CHF}$ and therefore we cannot automatically assume that what happens in CHF also happens in diseases that are not classically considered to have such high levels of neuroendocrine activation as CHF. This applies, for example, to type 2 diabetes mellitus. We therefore set out to see whether gradual reactivation of vascular tissue angiotensin I (AI) to AII conversion does indeed occur during chronic ACE inhibitor therapy in the kind of type 2 diabetic patients treated in micro HOPE. If vascular tissue AI to AII conversion did gradually reactivate over time in people with diabetes mellitus, it could in theory have implications for whether it might be better to use angiotensin receptor blockers (ARB), either instead of or together with ACE inhibitors in diabetic patients.

\section{Methods}

We used differential intrabrachial artery infusion of AI and AII to measure vascular tissue conversion of AI into AII. This is a well-established technique based on the premise that AI only produces vasoconstriction when converted into AII in vascular tissue [9]. There are two reasons why this technique is thought to mainly reflect $\mathrm{AI}$ to AII conversion in vascular tissue, rather than within the circulation [11]. The first reason is that AI to AII conversion in blood is much slower than the transit time in the circulation. The second reason is that the offset of the effect of intra-arterial enalaprilat on $\mathrm{AI}$ responses is prolonged with a half-life that is 16 times longer than the transit time in the forearm circulation and hence 16 times longer than the time enalaprilat will be present in the blood locally.
A total of 30 patients (mean age $43.5 \pm 10.8$ years $[ \pm \mathrm{SD}]$; 18 men, 14 women) with type 2 diabetes mellitus were studied. They all gave written informed consent and the study was approved by our local research ethics committee. They had either previously experienced a cardiovascular event or one other concurrent major cardiovascular risk factor in the absence of heart failure. They were treated with lisinopril, $20 \mathrm{mg}$ daily, over 18 months. Lisinopril was used because this was the ACE inhibitor most commonly used in the UK at the time the study began. The dose chosen $(20 \mathrm{mg})$ is, according to the British National Formulary (BNF), the usual maintenance dose in hypertension. The BNF also recommends a usual dose range for lisinopril of $10-20 \mathrm{mg}$ in patients with diabetic nephropathy.

The average duration of diabetes among our subjects was $7 \pm 3.1$ years with a mean $\mathrm{HbA}_{1 \mathrm{c}}$ of $7.2 \pm 0.7 \%$. Vascular tissue AI to AII conversion was studied using sequential infusions of AI (16, 64 and $256 \mathrm{pmol} / \mathrm{min})$ and AII (4, 16 and $64 \mathrm{pmol} / \mathrm{min}$ ) into the non-dominant brachial artery, with a saline washout period between the two infusions. Responses were assessed by forearm venous occlusion plethysmography at baseline, 9 and 18 months, following an initial 2 month run-in period. Full details of our protocol have been published before [9]. For each study, venous plasma RAAS activity markers (AI, AII, ACE, renin activity, aldosterone) were collected, centrifuged, stored and analysed by previously described methods [9]. Statistical analysis was performed using two-way analysis of variance with repeated measures.

\section{Results}

The baseline characteristics of our patient group are shown in Table 1. The only difference from contemporary treatment is an under-use of statins, but our use of statins was in fact similar to the HOPE study [1]. In both cases, this under-use was due to the fact that these studies began many years ago. Nevertheless, total cholesterol in our study was controlled to below the $5 \mathrm{mmol} / \mathrm{l}$ target that existed when this study began. The plasma levels of renin activity, AI, AII and aldosterone are shown in Figs. 1 and 2. The high levels of plasma AI and low levels of plasma AII show that the patients were indeed taking chronic ACE inhibitory therapy. This is confirmed by plasma ACE levels remaining below our assay detection limit $(<5 \mathrm{IU} / \mathrm{l})$ at all three timepoints. No significant alterations in the plasma levels of any of these RAAS components were seen during the 18 months of study. This suggests that the patients were compliant with their chronic lisinopril therapy. $\mathrm{HbA}_{\mathrm{lc}}$ was stable throughout (7.2, 7.0 and 7.0\% at the three time-points).

However, the results for vascular tissue RAAS were very different from the plasma RAAS results. The baseline 
Table 1 Baseline characteristics of study subjects

Group characteristics $(n=30)$

\begin{tabular}{ll}
\hline Age, years (range) & $43.5 \pm 5.4(32-71)$ \\
Men, $n$ (\%) & $18(60)$ \\
Women, $n$ (\%) & $12(40)$ \\
Smoking history & \\
Never, $n$ & 16 \\
Previous, $n$ & 9 \\
Present, $n$ & 5 \\
Ischaemic heart disease, $n$ & 23 \\
Hypertension, $n$ & 30 \\
Mean blood pressure, mmHg & $128 / 71 \pm 6 / 3$ \\
Mean total cholesterol, mmol/1 & $4.2 \pm 1.2$ \\
Mean duration of type 2 diabetes, years & $7 \pm 1.6$ \\
Mean HbA ${ }_{1 \mathrm{l}}, \%$ & $7.2 \pm 0.35$ \\
Mean dose of ACE inhibitor, mg/day & 20 \\
Mean duration of ACE inhibition, years & $4.3 \pm 2.1$ \\
Concomitant medication & \\
Statins, $n$ (mean equivalent & $14(22.1)$ \\
$\quad$ simvastatin dose, mg) & \\
Beta blockers, $n$ (mean equivalent & $13(5.0)$ \\
bisoprolol dose, mg) & \\
Diet control, \% & 4 \\
Gliclazide, $n$ (mean dose, mg) & $16(120)$ \\
Metformin, $n$ (mean dose, g) & $15(1.5)$ \\
Insulin, $n$ (mean dose, total daily units) & $10(64$ units) \\
\hline
\end{tabular}

Values are means $\pm \mathrm{SD}$, per cent or numbers $(n)$ as indicated

forearm blood flows (FBF) were not significantly different on the three study days. However, the AI-induced reduction in FBF was significantly greater $(p=0.01)$ at 18 months than at 0 months (Fig. 3). The percentage change in FBF response to $\mathrm{AI}$ infusion was $61 \%$ greater at 18 months than at baseline. The results at 9 months were intermediate between the 0 and 18 months results. By contrast, there were no significant changes in FBF responses to infused AII over the same 18 month study period ( $p=0.326$; Fig. 4). The results for infused AII imply that there was no significant upregulation or downregulation of vascular AII receptors during the 18 months of study. This allows us to conclude that the gradual increase in response to infused AI was indeed due to increased vascular AI to AII conversion, and not due to a change in vascular AII responses as such.

\section{Discussion}

We have clearly shown that during chronic lisinopril therapy in patients with diabetes, gradual reactivation of the vascular conversion of AI to AII occurs. Our other main finding was the dissociation between plasma RAAS and vascular tissue RAAS, shown by the fact that only the latter reactivated in this study.
Our study has two major strengths. First, we performed an in vivo study in humans. Hence there are no concerns about species differences as there would be with animal studies, nor are there any concerns that the experimental model itself introduced artefacts, as might happen with ex vivo studies or in vitro studies. Second, we performed a longitudinal study of the same individuals. Often longitudinal phenomena are studied by using a cross-sectional approach, where many different individuals at different stages of their disease are each studied once. Such a crosssectional approach is an imperfect, if not flawed way to study longitudinal phenomena, because it introduces interindividual variation, which may over time swamp subtle yet important intra-individual changes.

An intriguing question for the future is whether the ACE genotype has any influence on whether an individual reactivates his or her vascular RAAS system or not. Our study was not powered to answer this question, because a genotype study with three genotypes in only 30 individuals would be absurdly small and could lead to erroneous conclusions. However, the raw data for this study do not suggest that there were major inter-individual differences in the amount of reactivation seen. Nevertheless, this is an issue that needs to be studied more closely in the future, rather than being addressed by this study, which was too small.

Another intriguing question is whether the reactivation seen by us would also occur at higher lisinopril doses. We deliberately chose a dose of lisinopril that is regarded by the

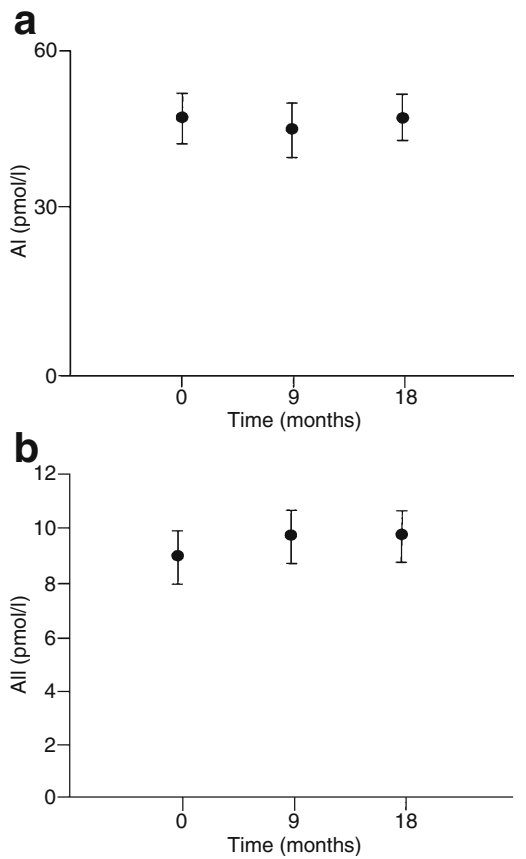

Fig. 1 Plasma AI (a) and AII (b) levels during 18 months of chronic lisinopril therapy $(20 \mathrm{mg}$ /day) in 30 type 2 diabetic patients. No significant changes occurred 


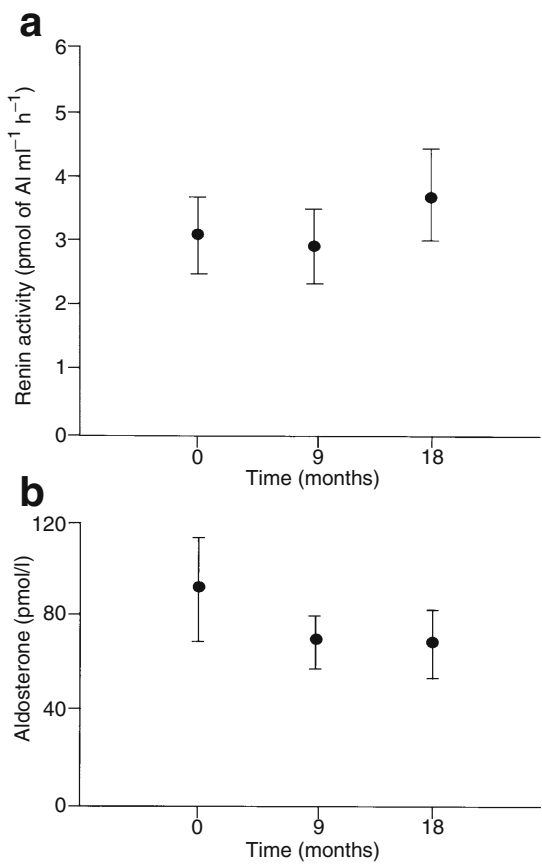

Fig. 2 Plasma renin activity (a) and aldosterone (b) levels during 18 months of chronic lisinopril therapy (20 mg/day) in 30 type 2 diabetic patients. No significant changes occurred

BNF as being at the top of the usual recommended maintenance dose. So whatever we found would be directly relevant to everyday clinical practice for the majority of patients. Another unknown is whether other ACE inhibitors, which are more tissue-bound, would demonstrate the same effect. However, again we decided to use the commonest ACE inhibitor in use in the UK, thus making our study relevant to the majority of UK patients.

One obvious unanswered question is the nature of the mechanism, whereby AI to AII conversion reactivates during chronic ACE inhibitor therapy. It could be due to reactivation of vascular ACE itself or to other non-ACE enzymes such as chymase. In $\mathrm{CHF}$, there is evidence that both probably contribute [8-10]. Two studies in CHF suggest that vascular ACE itself contributes, because in those studies increases of the ACE inhibitor dose re-established some further suppression of vascular tissue AI to AII conversion. On the other hand, one other study in CHF demonstrates that vascular tissue AII can indeed be generated by non-ACE pathways during chronic ACE inhibitor therapy [8-10]. To date, there are no data on this mechanistic question in nonCHF patients. Recent new insights in angiotensin research do not offer any simple alternative explanations for our finding [12]. For example, AI accumulates during ACE inhibitor therapy and some of that becomes the vasodilator, angiotensin (1-7), but there is no apparent reason why less AI should become angiotensin (1-7) during chronic ACE inhibitor therapy, unless more AI is channelled into AII instead, which is what we are proposing anyway.

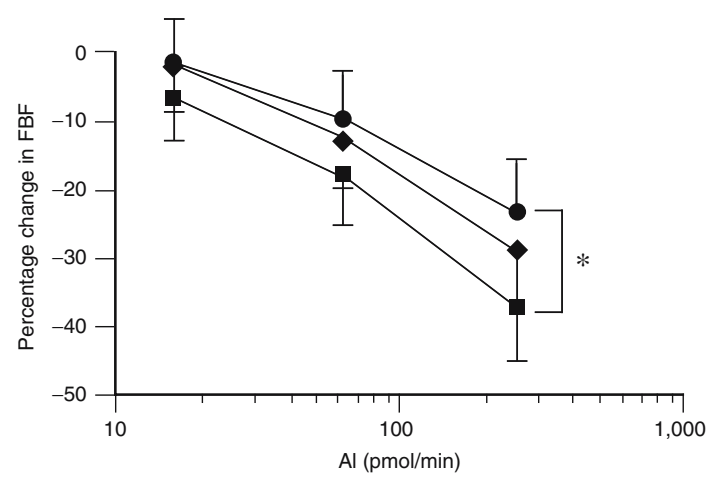

Fig. $3 \mathrm{FBF}$ responses to AI infusion at baseline (black circles), 9 (black diamonds) and 18 (black squares) months. ${ }^{*} p=0.01$

A question that follows from the above is why vascular ACE might reactivate (if it does) during chronic ACE inhibitor therapy. Again there are two possibilities. First, in tissue culture captopril was found to induce ACE in human endothelial cells [13]. This is akin to the phenomenon of upregulation of receptors when they are exposed constantly to an antagonist drug. The second possibility is that progressive atherosclerosis in the vasculature induces ACE activity [14-16]. However, overt atherosclerosis is not prominent in brachial arteries, although there may be enough progressive vascular/endothelial dysfunction to induce local ACE activity. Further work would be required to clarify these issues more precisely.

Our study shows a dissociation between the plasma RAAS components and vascular tissue RAAS in that only the latter reactivated over time. In fact, this mirrors exactly what we found in our previous study in CHF, emphasising again the dissociation between plasma and tissue components of the RAAS [9]. The reasons for this dissociation are not at all clear, since plasma AII is now thought to be

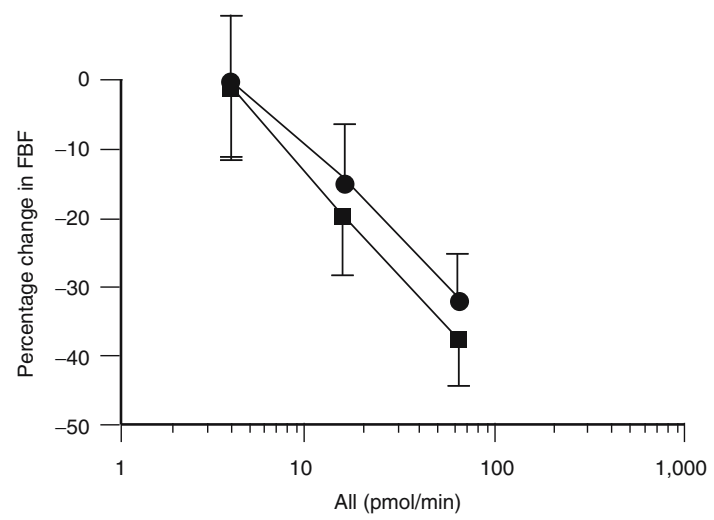

Fig. $4 \mathrm{FBF}$ responses to AII infusion at baseline (black circles), 9 (black diamonds) and 18 (black squares) months. $p=0.326$. Please note: the 9 month data overlap the 18 month data and so this line is not visible 
derived to some extent from the tissues, from vascular ACE and from kidney-derived renin.

Do our data have any clinical implications? If a similar phenomenon occurs in renal tissue, then our results could provide a mechanistic rationale to explain why a combined $\mathrm{ACE}$ inhibitor and ARB therapy performs better in diabetic microalbuminuria $[17,18]$. Second, and more speculatively, is the possibility that the gradual reactivation of vascular tissue AI to AII conversion during chronic ACE inhibitor therapy could detract from the cardioprotective benefits achieved when ACE inhibitors are used chronically, as in HOPE study type diabetic patients. Partial support for this possibility comes from the CHF patients enrolled in the Candesartan in Heart Failure: Assessment of Reduction in Mortality and Morbidity Added Trial [19], where adding candesartan to an ACE inhibitor produced further reductions in cardiovascular deaths and CHF hospitalisations. However, this support is only partial because other studies such as the Valsartan in Acute Myocardial Infarction Trial (VALIANT) did not make the same findings, possibly because the ACE inhibitor dose was halved when it was combined with an ARB in VALIANT [20]. This was done for safety reasons, but it does limit the ability of the VALIANT trial to inform us about the clinical consequences of adding an ARB to an ACE inhibitor. Nevertheless, it should be noted that in the VALIANT trial the ARB-alone arm did no better than the ACE inhibitor-alone arm, even though it could be expected to do so if ACE reactivation really does detract from the ACE inhibitor-alone benefit. Moreover, the kind of information seen in CHF studies cannot automatically be extrapolated to other non-CHF disease states. In fact, large ongoing studies are currently addressing whether or not an ARB produces added benefit in HOPE study type patients without heart failure.

One limitation of our study is that we did not study a nondiabetic, non-CHF control group in order to see if AI to AII conversion reactivation only occurs in diabetic and CHF patients. In fact, there is no reason to believe that this phenomenon should be limited to these two patient subgroups, but future work would be required to examine this.

In conclusion, we have clearly shown for the first time that vascular AI to AII conversion does gradually increase over time in vivo in humans with type 2 diabetes mellitus. Further studies are required to elucidate the precise mechanism of this gradual reactivation and whether overcoming this phenomenon will enhance the clinical benefits of chronic ACE inhibitor therapy in HOPE study type patients with diabetes mellitus. Possible ways to overcome this phenomenon are either to increase the ACE inhibitor dose to above current target doses or to add an ARB to ACE inhibitor therapy.

Acknowledgements We thank the British Heart Foundation for their financial support.
Duality of interest The authors declare that there is no duality of interest associated with this manuscript.

\section{References}

1. Yusuf S, Sleight P, Pogue J, Bosch J, Davies R, Dagenais G (2000) Effects of an ACE inhibitor, ramipril, on cardiovascular events in high risk patients. The Heart Outcomes Prevention Evaluation Study Investigators. N Engl J Med 342:145-150

2. Heart Outcomes Prevention Evaluation (HOPE) Study Investigators (2000) Effects of ramipril on cardiovascular and microvascular outcomes in people with diabetes: results of the HOPE and MICROHOPE substudy. Lancet 355:253-259

3. Lindholm LH, Ibsen H, Dahlof B et al LIFE Study Group (2002) Cardiovascular morbidity and mortality in patients with diabetes in the Losartan Intervention For Endpoint reduction in hypertension study (LIFE): a randomised trial against atenolol. Lancet 359:1004-1010

4. MacFadyen RJ, Lee AFC, Morton JJ, Pringle SD, Struthers AD (1999) How often are angiotensin II and aldosterone raised during chronic ACEI therapy in cardiac failure? Heart 82:57-61

5. Hirsch AT, Talsness CE, Schunkert H, Paul M, Dzau VJ (1991) Tissue specific activation of cardiac ACE in experimental heart failure. Circ Res 69:475-484

6. Okamura T, Miyazcki M, Inagenui T, Toad V (1986) Vascular RAS in 2 kidney, 1 clip hypertensive rates. Hypertension 8:560-565

7. Massie BM, Kramer BL, Topic N (1984) Lack of relationship between short term haemodynamic effects of captopril and subsequent clinical responses. Circulation 69:1135-1141

8. Petrie MC, Padmanabhan N, McDonald JE, Hillier C, Connell JMC, McMurray JJ (2001) ACE and non-ACE dependent AII generation in resistance arteries from patients with heart failure and coronary heart disease. J Am Coll Cardiol 37:1056-1061

9. Farquharson CAJ, Struthers AD (2002) Gradual reactivation over time of vascular AI to AII conversion during chronic lisinopril therapy in patients with chronic heart failure. J Am Coll Cardiol 39:767-775

10. Jorde U, Ennezat PV, Lisker J et al (2000) Maximally recommended doses of ACE inhibitors do not completely prevent ACE mediated formation of AII in CHF. Circulation 101:844-846

11. Benjamin N, Cockcroft JR, Collier JG, Dollery CT, Ritter JM, Webb DJ (1989) Local inhibition of converting enzyme and vascular responses to angiotensin and bradykinin in human forearm. J Physiol 412:543-555

12. Ferrario CM, Trask AJ, Jessup JA (2005) Advances in biochemical and functional roles of ACE2 and angiotensin (1-7) in regulation of cardiovascular function. Am J Physiol Heart Circ Physiol 289:H2281-H2290

13. Fyhroquist F, Hortling L, Gronhagen-Riska C (1982) Induction of ACE by captopril in cultured human endothelial cells. J Clin Endocrinol Metab 55:783-786

14. Fukuhara M, Geary RL, Diz DI et al (2000) Angiotensinconverting enzyme expression in human carotid artery atherosclerosis. Hypertension 35:353-359

15. Hoshida S, Kato J, Nishino M et al (2001) Increased angiotensinconverting enzyme activity in coronary artery specimens from patients with acute coronary syndrome. Circulation 103:630-633

16. Rakugi H, Kim DK, Krieger JE, Wang DS, Dzau VJ, Pratt RE (1994) Induction of angiotensin converting enzyme in the neointima after vascular injury. Possible role in restenosis. J Clin Invest 93:339-346

17. Mogensen CE, Neldam S, Tikkanen I et al (2000) Randomised controlled trial of dual blockade of renin-angiotensin system in 
patients with hypertension, microalbuminuria and non insulin dependent diabetes: the candesartan and lisinopril microalbuminuria (CALM) study. BMJ 321:1440-1444

18. Nakao N, Yoshimura A, Morita H, Takada M, Kayano T, Ideura T (2003) Combination therapy of angiotensin II receptor blocker and ACEI in non diabetic renal disease (COOPERATE): a randomised controlled trial. Lancet 361:117-124
19. McMurray JJV, Ostergren J, Swedberg K et al for CHARM investigators (2003) Effects of candesartan in patients with CHF and reduced LV systolic function taking ACE inhibitors: the CHARM added trial. Lancet 362:767-771

20. Pfeffer MA, McMurray JJ, Velazquez EJ et al (2003) Valsartan, captopril, or both in myocardial infarction complicated by heart failure, left ventricular dysfunction or both. N Engl J Med 349(20):1893-1906 